\title{
Restauração de dentes tratados endodonticamente com resinas bulk-fill: revisão integrativa
}

\author{
Endodontically treated teeth restored with bulk-fill resins: \\ integrative review
}

\author{
Viviane França* \\ Fernando Freitas Portella* \\ Eduardo Galia Reston ${ }^{* * *}$ \\ Guilherme Anziliero Arossi****
}

\section{Resumo}

Objetivo: realizar uma revisão de literatura sobre o uso de resinas bulk-fill para restaurar dentes tratados endodonticamente. Materiais e método: a busca dos estudos, clínicos ou laboratoriais, foi realizada no portal PubMed, utilizando os descritores "bulk fill" e "endodontically treated". Os dados extraídos da literatura foram agrupados em um quadro, que apresenta as características metodológicas e os principais resultados de cada estudo. Resultados: Sete estudos foram incluídos na revisão. Dentre eles, cinco avaliaram a resistência à fratura dos dentes após a restauração, um avaliou a adaptação da resina ao assoalho da câmara pulpar, e um realizou um ensaio clínico com acompanhamento de três anos, comparando o uso de resina bulk-fill com resina convencional. Considerações finais: o desempenho das resinas bulk-fill mostrou-se semelhante ao das resinas convencionais nas características de resistência à fratura dos dentes (in vitro) e longevidade (in vivo). A economia de tempo clínico proporcionada pelas resinas bulk-fill pode justificar o seu emprego para a restauração de dentes tratados endodonticamente.

Palavras-chave: Cavidade pulpar. Dente não vital. Restauração dentária permanente.

\section{Introdução}

Nas últimas décadas, as resinas compostas passaram a ter um papel importantíssimo na Odontologia, uma vez que os dentes restaurados com esse tipo de material apresentam longevidade adequada, estética satisfatória, além da praticidade de permitir a restauração direta dos dentes em uma única consulta. Apresentando menor tempo de trabalho e técnica de aplicação incremental, ao longo do tempo, as resinas compostas substituíram o amálgama de prata $^{1,2}$ e têm sido o material mais usado nas restaurações diretas. ${ }^{1}$

A demanda por materiais e técnicas que requeiram um tempo de aplicação reduzido, resultando em melhor custo-efetividade nos tratamentos, fez com que surgissem as resinas denominadas bulk-fill. Essas resinas dispensam a realização da técnica incremental, uma vez que podem ser aplicadas em incrementos únicos de até $4 \mathrm{~mm},{ }^{3-5}$ contrariamente às resinas convencionais. ${ }^{6}$ Teoricamente, nas resinas bulk-fill, a tensão de contração de polimerização é reduzida, quando comparada às resinas tradicionais. Para que a conversão polimérica não seja afetada, essas resinas apresentam maior translucidez, facilitando a passagem da luz e a sensibilização do sistema iniciador, o que contribui para uma adequada polimerização. ${ }^{5}$

Uma revisão sistemática de estudos clínicos mostrou que as resinas bulk-fill apresentam desempenho clínico semelhante às resinas tradicionais, 
quando utilizadas para restaurações diretas (classe I e II) em dentes posteriores. ${ }^{7}$ Dessa forma, para reabilitação de dentes sem grandes destruições coronárias, as resinas bulk-fill são uma opção para o profissional. O emprego das resinas bulk-fill na confecção de restaurações diretas representa uma simplificação da técnica, tornando-a menos sensível, haja vista que o profissional não necessita controlar o volume de diversos incrementos do material. $\mathrm{O}$ emprego dessas novas resinas permite economia de tempo clínico sem prejudicar a longevidade do tratamento restaurador. ${ }^{7}$

Em situações em que há extensas perdas estruturais, como no caso de dentes tratados endodonticamente, o emprego das resinas bulk-fill é uma alternativa promissora. A técnica de inserção em incremento único favorece a realização do procedimento restaurador imediatamente após a conclusão do tratamento endodôntico, evitando que os dentes permaneçam com restaurações provisórias e consequentemente eliminando os riscos de recontaminação dos sistemas de canais radiculares ${ }^{8,9}$ e fraturas coronárias até a reabilitação do elemento dental. ${ }^{10,11}$ Dessa forma, o objetivo deste estudo foi realizar uma revisão de literatura sobre as resinas bulk-fill empregadas para restauração de dentes tratados endodonticamente.

\section{Materiais e método}

A síntese dos estudos que avaliam o emprego de resina bulk-fill em procedimentos para reabilitação de dentes tratados endodonticamente foi realizada a partir de uma revisão integrativa da literatura. A busca dos estudos foi realizada no portal PubMed, utilizando as palavras-chave: "bulk fill" AND "endodontically treated”. Nenhuma restrição quanto ao idioma ou à data de publicação dos artigos foi empregada. Foram incluídos estudos clínicos e laboratoriais que avaliaram as resinas bulk-fill emprega- das para restauração de dentes tratados endodonticamente, usando a técnica de incremento único, com ou sem cobertura oclusal em resina convencional. Estudos que empregaram resinas bulk-fill sem utilizar técnica incremental foram excluídos da revisão, assim como aqueles em que as resinas bulk-fill não foram utilizadas para restaurações do tipo diretas.

Dois dos pesquisadores autores deste estudo (V.F. e F.F.P.) fizeram a leitura completa dos artigos e realizaram a extração dos dados. Foram coletadas as informações: título do artigo, ano de publicação, nome do primeiro autor, objetivo do estudo, tipo de estudo, descrição dos métodos empregados e principais resultados. Os dados extraídos foram agrupados em um quadro.

\section{Resultados}

A partir da estratégia de busca adotada, retornaram 13 artigos. Após a leitura completa dos artigos, sete foram incluídos. Dentre os estudos incluídos, cinco avaliaram a resistência à fratura dos dentes após a restauração, um avaliou a adaptação da resina ao assoalho da câmara pulpar, e um realizou um ensaio clínico, com acompanhamento de três anos, comparando o uso de resina bulk-fill com resina convencional. O Quadro 1 mostra os trabalhos incluídos.

As razões para exclusão dos demais artigos foram: utilização da resina bulk-fill para confecção de núcleo de preenchimento; ${ }^{12,13}$ aplicação do material como barreira intracanal; ${ }^{14}$ utilização da resina para reforçar internamente canal muito amplo; ${ }^{15}$ aplicação do material usando técnica incremental:16 e avaliação de corpos de prova da resina exclusivamente, sem nenhuma aplicação direta em dentes. ${ }^{17}$ 
Quadro 1 - Descrição dos estudos avaliando o emprego de resinas bulk-fill na restauração de dentes tratados endodonticamente

\begin{tabular}{|c|c|c|c|c|c|}
\hline $\begin{array}{l}\text { Primeiro autor e ano de } \\
\text { publicação }\end{array}$ & Objetivos do estudo & $\begin{array}{l}\text { Tipo de estudo e } \\
\text { dentes utilizados }\end{array}$ & Métodos empregados & Grupos de comparação & Resultados/Conclusão \\
\hline Scotti et al..$^{18}(2016)$ & $\begin{array}{l}\text { Avaliar a adaptação de resinas } \\
\text { compostas ao assoalho da } \\
\text { câmara pulpar }\end{array}$ & $\begin{array}{l}\text { In vitro, com molares } \\
\text { humanos extraidos }\end{array}$ & $\begin{array}{l}\text { Após o tratamento endodôntico, realizou-se } \\
\text { o selamento da embocadura dos canais e do } \\
\text { assoalho da câmara pulpar com diferentes } \\
\text { resinas compostas. } \\
\text { Avaliou-se a adaptação dos materiais restau- } \\
\text { radores por tomografia óptica após seccionar } \\
\text { os dentes longitudinalmente. } \\
\text { Aferiu-se a distância média do material res- } \\
\text { taurador à dentina do assoalho da câmara } \\
\text { pulpar ao longo de toda a interface adesiva. }\end{array}$ & $\begin{array}{l}\text { A: camada horizontal de } 0,5 \mathrm{~mm} \text { de resina } \\
\text { convencional flow + restauração com resina } \\
\text { convencional utilizando técnica incremental; } \\
\text { B: restauração completa com resina bulk- } \\
\text {-fill flow; } \\
\text { C: restauração completa com resina con- } \\
\text { vencional utilizando técnica incremental. }\end{array}$ & $\begin{array}{l}\text { A adaptação das resinas convencional } \\
\text { flow e bulk-fill flow não diferem entre } \\
\text { si, ambas apresentam melhor adapta- } \\
\text { ção que a resina convencional. }\end{array}$ \\
\hline Karaman et al. ${ }^{19}(2017)$ & $\begin{array}{l}\text { Avaliar a longevidade de res- } \\
\text { taurações classe II em dentes } \\
\text { posteriores confeccionadas } \\
\text { com diferentes técnicas (incre- } \\
\text { mental ou incremento único) } \\
\text { e diferentes materiais (resina } \\
\text { composta convencional ou } \\
\text { bulk-fill) }\end{array}$ & $\begin{array}{l}\text { In vivo, em ensaio } \\
\text { clínico randomizado }\end{array}$ & $\begin{array}{l}\text { Foi realizado trabalho clínico com tempo de } \\
\text { acompanhamento de } 3 \text { anos. } \\
\text { Usaram-se como controle dentes do próprio } \\
\text { paciente. } \\
\text { Foram confeccionadas } 47 \text { restaurações clas- } \\
\text { se Il (OD ou MO), em pré-molares ou molares, } \\
\text { em } 37 \text { pacientes. } \\
\text { Houve reavaliação em } 6 \text { meses, } 1,2 \text { e } 3 \text { anos, } \\
\text { de acordo com o critério USPHS modificado. } \\
\text { Consideraram-se retenção, pigmentação mar- } \\
\text { ginal, adaptação marginal, cor, textura superfi- } \\
\text { cial, anatomia, cárie secundária. }\end{array}$ & $\begin{array}{l}\text { A: camada de } 2 \mathrm{~mm} \text { de resina convencio- } \\
\text { nal flow + resina convencional em técnica } \\
\text { incremental; } \\
\text { B: resina bulk-fill flow em incremento de } \\
4 \mathrm{~mm} \text { e resina convencional em oclusais } \\
2 \mathrm{~mm} \text {. }\end{array}$ & $\begin{array}{l}\text { A longevidade das restaurações não } \\
\text { diferiu entre os grupos para nenhum } \\
\text { dos critérios de avaliação. }\end{array}$ \\
\hline Tekçe et al..$^{20}(2017)$ & 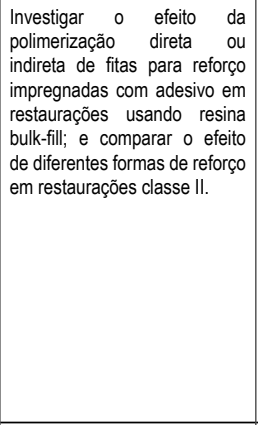 & $\begin{array}{l}\text { In vitro, com molares } \\
\text { humanos extraídos }\end{array}$ & $\begin{array}{l}\text { Após o tratamento endodôntico, foram reali- } \\
\text { zados preparos cavitários classe II (MOD), e } \\
\text { as caixas proximais, seladas com resina com- } \\
\text { posta convencional. } \\
\text { Os dentes foram restaurados com diferentes } \\
\text { materiais, incluindo resina bulk-fill e fibras de } \\
\text { reforço, e protocolos de fotoativação e subme- } \\
\text { tidos ao ensaio de resistência à fratura, sendo } \\
\text { aplicada uma carga compressiva no sentido } \\
\text { vertical. }\end{array}$ & $\begin{array}{l}\text { A: reforço com fita (fotoativação imediata) } \\
\text { + aplicação de resina bulk-fill flow }(4 \mathrm{~mm} \text { ) + } \\
\text { resina convencional; } \\
\text { B: reforço com fita + aplicação de resina } \\
\text { bulk-fill flow (4 mm) (fotoativação do con- } \\
\text { junto fita + resina bulk-fill) + resina conven- } \\
\text { cional; } \\
\text { C: reforço com fita (fotoativação imediata) } \\
\text { + aplicação de resina convencional flow } \\
(2 \mathrm{~mm})+\text { resina convencional; } \\
\text { D: reforço com fita (fotoativação imediata) + } \\
\text { aplicação de resina convencional em técnica } \\
\text { incremental; } \\
\text { E: aplicação de resina reforçada por fibra } \\
(3 \mathrm{~mm})+\text { resina convencional; } \\
\text { F: cavidade sem restauração; } \\
\text { G: dente hígido. }\end{array}$ & $\begin{array}{l}\text { Todos os tratamentos aumentaram } \\
\text { a resistência do dente, quando com- } \\
\text { parados ao grupo sem restauração. } \\
\text { Não há diferença entre os diferentes } \\
\text { tratamentos. }\end{array}$ \\
\hline Yasa et al. ${ }^{21}(2016)$ & $\begin{array}{|lr|}\text { Avaliar a resistência à } \\
\text { fratura de dentes } & \text { tratados } \\
\text { endodonticamente } & \text { e } \\
\text { restaurados com } & \text { diferentes } \\
\text { resinas compostas e diferentes } \\
\text { preparos cavitários, com e sem } \\
\text { slot de retenção }\end{array}$ & $\begin{array}{l}\text { In vitro, com molares } \\
\text { humanos extraídos. }\end{array}$ & $\begin{array}{l}\text { Após o tratamento endodôntico, foram reali- } \\
\text { zados preparos classe II (MOD) com ou sem } \\
\text { slot de retenção. } \\
\text { Os dentes foram restaurados com diferentes } \\
\text { materiais e submetidos ao ensaio de resis- } \\
\text { tência à fratura, sendo aplicada uma carga } \\
\text { compressiva no sentido vertical. }\end{array}$ & $\begin{array}{l}\text { A: sem restauração (com e sem slot); } \\
\text { B: resina convencional usando técnica incre- } \\
\text { mental (com e sem slot); } \\
\text { C: resina bulk-fill flow (4 mm) + resina con- } \\
\text { vencional; } \\
\text { D: resina reforçada por fibra de vidro }(4 \mathrm{~mm}) \\
\text { + resina convencional. }\end{array}$ & $\begin{array}{l}\text { A aplicação de material restaurador } \\
\text { nos dentes, tanto com slot quanto } \\
\text { sem, aumenta a resistência do dente } \\
\text { não restaurado. } \\
\text { Não há diferença na resistência } \\
\text { dos dentes restaurados com resina } \\
\text { convencional ou com bulk-fill. }\end{array}$ \\
\hline Isufi et al. ${ }^{22}$ (2016) & $\begin{array}{|lr|}\text { Comparar a resistência à } \\
\text { fratura de dentes } & \text { tratados } \\
\text { endodonticamente } & e \\
\text { restaurados com resina bulk-fill } \\
\text { ou convencional. }\end{array}$ & $\begin{array}{l}\text { In vitro, com molares } \\
\text { humanos extraídos }\end{array}$ & $\begin{array}{l}\text { Após o tratamento endodôntico de molares } \\
\text { superiores e inferiores, a cavidade de aces- } \\
\text { so (oclusal) foi restaurada com diferentes } \\
\text { materiais. } \\
\text { Os dentes foram restaurados com diferentes } \\
\text { materiais e submetidos ao ensaio de resis- } \\
\text { tência à fratura, sendo aplicada uma carga } \\
\text { compressiva em um ângulo de } 30^{\circ} \text { ao longo } \\
\text { do eixo do dente. }\end{array}$ & $\begin{array}{l}\text { A: dente hígido; } \\
\text { B: resina convencional (técnica incremen- } \\
\text { tal); } \\
\text { C: resina bulk-fill flow }(4 \mathrm{~mm})+\text { resina con- } \\
\text { vencional. }\end{array}$ & $\begin{array}{l}\text { Não houve diferença entre os grupos } \\
\text { quanto à resistência dos dentes. }\end{array}$ \\
\hline Atalay et al. ${ }^{23}(2016)$ & $\begin{array}{|lrr|}\text { Avaliar a } & \text { resistência } & \text { à } \\
\text { fratura de } & \text { dentes } & \text { tratados } \\
\text { endodonticamente } & e \\
\text { restaurados } & \text { com } & \text { diferentes } \\
\text { tipos de materiais. } & \end{array}$ & $\begin{array}{l}\text { In vitro, com pré- } \\
\text { molares humanos } \\
\text { extraídos }\end{array}$ & $\begin{array}{l}\text { Após o tratamento endodôntico, pré-molares } \\
\text { com preparos classe II (MOD) foram restau- } \\
\text { rados com diferentes materiais restauradores } \\
\text { diretos. } \\
\text { Para cada material restaurador, foram utiliza- } \\
\text { dos sistemas adesivos compativeis do mes- } \\
\text { mo fabricante. } \\
\text { Os dentes foram submetidos ao ensaio de re- } \\
\text { sistência à fratura, sendo aplicada uma carga } \\
\text { compressiva no sentido vertical. }\end{array}$ & $\begin{array}{l}\text { A: dente hígido; } \\
\text { B: dente sem restauração; } \\
\text { C: adesivo convencional de dois passos + } \\
\text { resina bulk-fill (incrementos de } 5 \mathrm{~mm} \text { ): fa- } \\
\text { bricante } 3 \mathrm{M} ; \\
\text { D: adesivo convencional de dois passos + } \\
\text { resina bulk-fill flow }(4 \mathrm{~mm})+\text { resina conven- } \\
\text { cional: fabricante Denstlply; } \\
\text { E: adesivo autocondicionante de um passo } \\
\text { + resina reforçada por fibra de vidro }(4 \mathrm{~mm}) \\
\text { + resina convencional: fabricante } \mathrm{GC} ; \\
\text { F: adesivo convencional de dois passos + } \\
\text { resina convencional (incrementos de } 2 \mathrm{~mm}) \text {. } \\
\end{array}$ & $\begin{array}{l}\text { Todos os tratamentos aumentaram } \\
\text { a resistência do dente, quando com- } \\
\text { parados ao grupo sem restauração. } \\
\text { Não há diferença entre os diferentes } \\
\text { tratamentos. }\end{array}$ \\
\hline Özyürek et al..$^{24}(2018)$ & $\begin{array}{l}\text { Comparar a resistência à } \\
\text { fratura de dentes tratados } \\
\text { endodonticamente, rom } \\
\text { diferentes formas de acesso } \\
\text { aos canais, e restaurados } \\
\text { com diferentes rateriais } \\
\text { restauradores. }\end{array}$ & $\begin{array}{l}\text { In vitro, com incisivos } \\
\text { inferiores humanos } \\
\text { extraídos }\end{array}$ & $\begin{array}{l}\text { Foram realizados tratamentos endodônticos } \\
\text { em molares inferiores utilizando duas formas } \\
\text { de acesso, tradicional e conservadora, envol- } \\
\text { vendo ambas a parede mesial do dente. } \\
\text { Os dentes foram restaurados com diferentes } \\
\text { materiais e submetidos ao ensaio de resis- } \\
\text { tência à fratura, sendo aplicada uma carga } \\
\text { compressiva em um ângulo de } 30^{\circ} \text { ao longo } \\
\text { eixo do dente. }\end{array}$ & $\begin{array}{l}\text { A: dente hígido; } \\
\text { B: acesso tradicional, restauração com re- } \\
\text { sina reforçada por fibra de vidro }(4 \mathrm{~mm})+ \\
\text { resina convencional; } \\
\text { C: acesso conservador, restauração com } \\
\text { resina reforçada por fibra de vidro }(4 \mathrm{~mm})+ \\
\text { resina convencional; } \\
\text { D: acesso tradicional, restauração com re- } \\
\text { sina bulk-fill }(4 \mathrm{~mm})+\text { resina convencional; } \\
\text { E: acesso conservador, restauração com } \\
\text { resina bulk-fill }(4 \mathrm{~mm})+\text { resina convencional; }\end{array}$ & $\begin{array}{l}\text { Os dentes restaurados com re- } \\
\text { sina bulk-fill apresentaram maior } \\
\text { resistência à fratura que os dentes } \\
\text { restaurados com resina reforçada com } \\
\text { fibra de vidro. } \\
\text { O tipo de acesso não influenciou a } \\
\text { resistência à fratura. }\end{array}$ \\
\hline
\end{tabular}




\section{Discussão}

A presente revisão, que teve como objetivo realizar uma revisão de literatura sobre as resinas bulk- fill empregadas para restauração de dentes tratados endodonticamente, aponta que o uso desse tipo de resina tem desempenho semelhante às resinas convencionais, quando utilizada em restaurações diretas. As resinas bulk-fill, desenvolvidas a partir da demanda dos profissionais por materiais restauradores diretos com menor sensibilidade técnica, já tiveram seu desempenho clínico avaliado em restaurações diretas de dentes sem grandes perdas estruturais, como os dentes que receberam tratamento de canal.

Nos trabalhos que avaliaram a resistência à fratura, na maior parte das vezes, eram comparados diversos grupos de materiais, alguns contando com preparos cavitários distintos. Em todos, as resinas bulk-fill apresentaram desempenho semelhante às resinas convencionais. Possivelmente a elevada translucidez, associada ao sistema de polimerização otimizado, fez com que, mesmo a resina sendo aplicada em grandes volumes, a polimerização fosse adequada, conferindo propriedades mecânicas satisfatórias. ${ }^{5,25}$ Além disso, embora haja tensões de contração distintas entre os tipos de resina e técnicas de inserção, ${ }^{17}$ essa variável não afetou a resistência à fratura dos dentes restaurados. ${ }^{20-24}$

A avaliação da adaptação das restaurações ao assoalho da câmara pulpar utilizando resinas bulk-fill flow não difere de uma resina convencional flow e é melhor que uma resina convencional. Isso se deve ao elevado escoamento dessas resinas, o que permite que, mesmo em regiões de difícil aplicação, como na entrada dos canais, o material restaurador entre em contato com todas as paredes dentinárias. ${ }^{18}$

O trabalho clínico incluído na revisão avaliou a longevidade das restaurações classe II por 3 anos, comparando as resinas bulk-fill com convencionais. Os autores avaliaram diversos parâmetros clínicos, como retenção, alteração de cor e rugosidade superficial, e não encontraram diferenças entre os grupos. Cabe ressaltar que, nos grupos restaurados com resina bulk-fill, o último incremento externo na face oclusal foi confecionado em resina convencional; contudo, na face proximal, a bulk-fill ficou exposta (técnica do sanduíche aberto). Considerando essa exposição da resina na face proximal, poderia se esperar um comportamento diferente, porém, a incidência de cárie secundária foi semelhante entre os grupos. ${ }^{19}$

Como limitações desta revisão, está o fato de a maior parte dos trabalhos tratar de estudos em laboratório e o único trabalho clínico incluído ter um tempo de acompanhamento relativamente curto, 3 anos. Dessa forma, a inserção definitiva das resinas bulk-fill para tratamento de dentes tratados endo- donticamente na prática clínica deve ser feita cautelosamente.

\section{Considerações finais}

O desempenho das resinas bulk-fill se mostrou semelhante às resinas convencionais, quando comparadas quanto à resistência à fratura dos dentes (in vitro) e à longevidade (in vivo). A economia de tempo clínico proporcionada pelas resinas bulk-fill encoraja o seu emprego para a restauração de dentes tratados endodonticamente.

\section{Abstract}

Objective: the aim of this study was to review the literature regarding the use of bulk-fill resin to restore endodontically treated teeth. Materials and method: the search was performed at PubMed, using the descriptors "bulk fill" AND "endodontically treated". Clinical and laboratorial studies were included. The extracted data was presented on a table showing the methodological features and results of each study. Results: seven studies evaluated the bulk-fill resin on direct restoration. Five of these seven evaluated the resistance to fracture, one assessed the resin adaptation to the floor chamber, and one was a randomized clinical trial comparing the bulkfill resin to conventional resin. Final considerations: the performance of bulk-fill resins was similar to the conventional resins when compared to the in vitro studies on tooth fracture resistance and in vivo study on longevity of restorations. The economy of clinical time may justify its use to restore endodontically treated teeth.

Keywords: Dental pulp cavity. Nonvital tooth. Dental restoration.

\section{Referências}

1. Demarco FF, Collares K, Correa MB, Cenci MS, Moraes RR, Opdam NJ. Should my composite restorations last forever? Why are they failing? Braz Oral Res 2017; 28(31):s1-e56.

2. Schwendicke F, Göstemeyer G, Blunck U, Paris S, Hsu LY, Tu YK. Directly placed restorative materials: review and network meta-analysis. J Dent Res 2016; 95(6):613-22.

3. Chesterman J, Jowett A, Gallacher A, Nixon P. Bulk-fill resin-based composite restorative materials: a review. Br Dent J 2017; 222(5):337-44

4. Maas MS, Alania Y, Natale LC, Rodrigues MC, Watts DC, Braga RR. Trends in restorative composites research: what is in the future? Braz Oral Res 2017; 28(31):s1-e55.

5. Reis AF, Vestphal M, Amaral RCD, Rodrigues JA, Roulet JF, Roscoe MG. Efficiency of polymerization of bulk-fill composite resins: a systematic review. Braz Oral Res 2017; 28(31):s1-e59.

6. Franco EB, Lopes LG. Contração de polimerização x adaptação marginal de restaurações em resina composta: abordagem atual. Ver Fac Odontol Univ Passo Fundo 2000; 5(1):37-41.

7. Veloso SRM, Lemos CAA, de Moraes SLD, do Egito Vasconcelos BC, Pellizzer EP, de Melo Monteiro GQ. Clinical performance of bulk-fill and conventional resin composite restora- 
tions in posterior teeth: a systematic review and meta-analysis. Clin Oral Investig 2018; Epub ahead of print.

8. Srivastava PK, Nagpal A, Setya G, Kumar S, Chaudhary A, Dhanker K. Assessment of coronal leakage of temporary restorations in root canal-treated teeth: an in vitro study. J Contemp Dent Pract 2017; 18(2):126-30.

9. Adnan S, Khan FR. Comparison of micro-leakage around temporary restorative materials placed in complex endodontic access cavities: an In-Vitro Study. J Coll Physicians Surg Pak 2016; 26(3):182-6.

10. Laustsen MH, Munksgaard EC, Reit C, Bjørndal L. A temporary filling material may cause cusp deflection, infractions and fractures in endodontically treated teeth. Int Endod J 2005; 38(9):653-7.

11. Milani AS, Froughreyhani M, Mohammadi H, Tabegh FG, Pournaghiazar F. The effect of temporary restorative materials on fracture resistance of endodontically treated teeth. Gen Dent 2016; 64(1):e1-4.

12. Jeaidi ZA. Fracture resistance of endodontically treated teeth restored with Zirconia filler containing composite core material and fiber posts. Pak J Med Sci 2016; 32(6):1474-8.

13. Lazari PC, de Carvalho MA, Del Bel Cury AA, Magne P. Survival of extensively damaged endodontically treated incisors restored with different types of posts-and-core foundation restoration material. J Prosthet Dent 2018; 119(5):769-76.

14. Yasa E, Arslan H, Yasa B, Akcay M, Alsancak M, Hatirli H. The force required to fracture endodontically roots restored with various materials as intra-orifice barriers. Niger J Clin Pract 2017; 20(10):1237-41.

15. Bakaus TE, Gruber YL, Reis A, Gomes OMM, Gomes GM. Bond strength values of fiberglass post to flared root canals reinforced with different materials. Braz Oral Res 2018; 1:32:e13.

16. Frankenberger R, Zeilinger I, Krech M, Mörig G, Naumann M, Braun A, et al. Stability of endodontically treated teeth with differently invasive restorations: adhesive vs. non-adhesive cusp stabilization. Dent Mater 2015; 31(11):1312-20.

17. Oliveira Schliebe LRS, Lourenço Braga SS, da Silva Pereira RA, Bicalho AA, Veríssimo C, Novais VR, et al. The new generation of conventional and bulk-fill composites do not reduce the shrinkage stress in endodontically-treated molars. Am J Dent 2016; 29(6):333-8.

18. Scotti N, Alovisi C, Comba A, Ventura G, Pasqualini D, Grignolo F, et al. Evaluation of composite adaptation to pulpal chamber floor using optical coherence tomography. J En$\operatorname{dod} 2016 ; 42(1): 160-3$.

19. Karaman E, Keskin B, Inan U. Three-year clinical evaluation of class II posterior composite restorations placed with different techniques and flowable composite linings in endodontically treated teeth. Clin Oral Invest 2017; 21(2):709-16.

20. Tekçe N, Pala K, Tuncer S, Demirci M, Serim ME. Influence of polymerisation method and type of fibre on fracture strength of endodontically treated teeth. Aust Endod J 2017; 43(3):115-22.

21. Yasa B, Arslan H, Yasa E, Akcay M, Hatirli H. Effect of novel restorative materials and retention slots on fracture resistance of endodontically-treated teeth. Acta Odontol Scand 2016; 74(2):96-102.

22. Isufi A, Plotino G, Grande NM, Ioppolo P, Testarelli L, Bedini $\mathrm{R}$, et al. Fracture resistance of endodontically treated teeth restored with a bulkfill flowable material and a resin composite. Ann Stomatol (Roma) 2016; 7(1-2):4-10.
23. Atalay C, Yazici AR, Horuztepe A, Nagas E, Ertan A, Ozgunaltay G. Fracture resistance of endodontically treated teeth restored with bulk fill, bulk fill flowable, fiber-reinforced, and conventional resin composite. Oper Dent 2016; 41(5):e131-40.

24. Özyürek T, Ülker Ö, Demiryürek EÖ, Yılmaz F. The effects of endodontic access cavity preparation design on the fracture strength of endodontically treated teeth: traditional versus conservative preparation. J Endod 2018; 44(5):800-5.

25. Shibasaki S, Takamizawa T, Nojiri K, Imai A, Tsujimoto A, Endo H, et al. Polymerization behavior and mechanical properties of high-viscosity bulk fill and low shrinkage resin composites. Oper Dent 2017; 42(6):e177-87.

\section{Endereço para correspondência:}

Fernando Freitas Portella

Universidade Luterana do Brasil, PPG Odontologia

Avenida Farroupilha, 8001, Prédio 59 (Odontologia), $3^{\circ}$ andar

92425-900, Canoas, RS, Brasil

Telefone: (+55) (51) 99235-1597

E-mail: portellaff@yahoo.com.br

Recebido: 06/08/18. Aceito: 20/08/18. 